\title{
Clinical trial demonstrates exercise following bariatric surgery improves insulin sensitivity
}

\author{
Paul M. Coen, ${ }^{1,2}$ Charles J. Tanner, ${ }^{3}$ Nicole L. Helbling, ${ }^{1}$ Gabriel S. Dubis, ${ }^{3}$ Kazanna C. Hames, ${ }^{1}$ Hui Xie, ${ }^{4}$ George M. Eid, ${ }^{5}$ \\ Maja Stefanovic-Racic, ${ }^{1}$ Frederico G.S. Toledo, ${ }^{1}$ John M. Jakicic, ${ }^{2}$ Joseph A. Houmard, ${ }^{3}$ and Bret H. Goodpaster ${ }^{1}$ \\ 'Division of Endocrinology and Metabolism, Department of Medicine, University of Pittsburgh, Pittsburgh, Pennsylvania, USA. Department of Health and Physical Activity, University of Pittsburgh, \\ Pittsburgh, Pennsylvania, USA. ${ }^{3}$ Department of Kinesiology, East Carolina University, Greenville, North Carolina, USA. ${ }^{4}$ Translational Research Institute for Metabolism and Diabetes, Florida Hospital, \\ Orlando, Florida, USA. D. Department of Surgery, University of Pittsburgh, Pittsburgh, Pennsylvania, USA
}

\begin{abstract}
BACKGROUND. Roux-en-Y gastric bypass (RYCB) surgery causes profound weight loss and improves insulin sensitivity (S) in obese patients. Regular exercise can also improve $S_{1}$ in obese individuals; however, it is unknown whether exercise and RYCB surgery-induced weight loss would additively improve $S_{1}$ and other cardiometabolic factors.
\end{abstract}

\begin{abstract}
METHODS. We conducted a single-blind, prospective, randomized trial with 128 men and women who recently underwent RYCB surgery (within 1-3 months). Participants were randomized to either a 6-month semi-supervised moderate exercise protocol ( $E X, n=66$ ) or a health education control (CON; $n=62$ ) intervention. Main outcomes measured included $S_{1}$ and glucose effectiveness $\left(S_{c}\right)$, which were determined from an intravenous glucose tolerance test and minimal modeling. Secondary outcomes measured were cardiorespiratory fitness $\left(\mathrm{VO}_{2}\right.$ peak) and body composition. Data were analyzed using an intention-to-treat (ITT) and per-protocol (PP) approach to assess the efficacy of the exercise intervention (>120 min of exercise/week).
\end{abstract}

RESULTS. 119 (93\%) participants completed the interventions, 95\% for CON and 91\% for EX. There was a significant decrease in body weight and fat mass for both groups $\left(P<0.001\right.$ for time effect). $S_{1}$ improved in both groups following the intervention (ITT: CON vs. EX; +1.64 vs. $+2.24 \mathrm{~min}^{-1} / \mu \mathrm{U} / \mathrm{ml}, P=0.18$ for $\Delta, P<0.001$ for time effect). A PP analysis revealed that exercise produced an additive $S_{1}$ improvement (PP: CON vs. EX; +1.57 vs. $+2.69 \mathrm{~min}^{-1} / \mu \mathrm{U} / \mathrm{ml}, P=0.019$ ) above that of surgery. Exercise also improved $S_{\mathrm{G}}$ (ITT: CON vs. EX; +0.0023 vs. $+0.0063 \mathrm{~min}^{-1}, P=0.009$ ) compared with the CON group. Exercise improved cardiorespiratory fitness ( $\mathrm{VO}_{2}$ peak) compared with the $\mathrm{CON}$ group.

CONCLUSION. Moderate exercise following RYGB surgery provides additional improvements in $\mathrm{S}_{1}, \mathrm{~S}_{\mathrm{G}}$, and cardiorespiratory fitness compared with a sedentary lifestyle during similar weight loss.

TRIAL REGISTRATION. clinicaltrials.gov identifier: NCT00692367.

FUNDING. This study was funded by the NIH/National Institute of Diabetes and Digestive and Kidney Diseases (R01 DK078192) and an NIH/National Center for Research Resources/Clinical and Translational Science Award (UL1 RR024153).

\section{Introduction}

Roux-en-Y gastric bypass (RYGB) is the most commonly performed metabolic surgery in the United States and results in dramatic weight loss and type 2 diabetes (T2D) remission in a large percentage of patients $(1,2)$. Although insulin resistance is a central component of T2D and its control is key to the prevention and treatment of T2D, improvements in insulin sensitivity $\left(\mathrm{S}_{\mathrm{I}}\right)$ in

Conflict of interest: J.M. Jakicic reports personal fees from Kaiser Permanente, outside the submitted work. G.M. Eid reports personal fees from Covidien, personal fees from power medical, and personal fees from Apollo Endosurgery, outside the submitted work. Role of funding source: The NIH had no role in the design and conduct of the study, the collection, management, analysis and interpretation of the data, or the preparation, review, or approval of the manuscript.

Submitted: July 14, 2014; Accepted: October 31, 2014.

Reference information: J Clin Invest. 2015;125(1):248-257. doi:10.1172/JCI78016. nondiabetic patients following RYGB surgery are typically quite modest compared with the presurgery condition (3). Moreover, there appear to be 2 discrete periods of improvement. The first is immediately after surgery, at which time hepatic, but not peripheral, $\mathrm{S}_{\mathrm{I}}$ improves in response to acute energy restriction (4-6), while greater, protracted weight loss appears to be more strongly associated with improved peripheral $S_{I}(7,8)$. Even with significant weight loss 1 year following RYBG surgery, peripheral $\mathrm{S}_{\mathrm{I}}$ is still low compared with that of lean metabolically healthy individuals $(3,5,6,9)$.

Exercise is considered a cornerstone for obesity treatment, and while it is not generally viewed to cause substantial body weight reduction (10), it can potently improve peripheral $S_{I}$ and glucose control (11-13) and can reduce the risk of T2D and cardiovascular disease $(14,15)$. There is general consensus that even a single session of moderate intensity exercise can induce an improvement in $S_{I}(16)$. There is also evidence that exercise can 


\section{Table 1. Baseline characteristics of study participants}

\begin{tabular}{|c|c|c|c|}
\hline Parameter & EX $(n=66)$ & $\operatorname{CON}(n=62)$ & $P$ value \\
\hline Age, mean yr (SD) & $41.3(9.7)$ & $41.9(10.3)$ & 0.69 \\
\hline \multicolumn{4}{|l|}{ Sex, ratio } \\
\hline Male/female & $7 / 59$ & $8 / 54$ & 0.69 \\
\hline \multicolumn{4}{|l|}{ Race, ratio } \\
\hline $\begin{array}{l}\text { African-American/mixed } \\
\text { European descent }\end{array}$ & $12 / 54$ & $10 / 52$ & 0.76 \\
\hline \multicolumn{4}{|l|}{ Anthropometrics, mean (SD) } \\
\hline Presurgery weight, kg & $127.2(22.6)$ & $121.8(25.7)$ & 0.25 \\
\hline Preintervention weight, $\mathrm{kg}$ & $107.3(19.9)$ & $105.7(25.1)$ & 0.71 \\
\hline Preintervention BMl, kg/m² & $38.8(6.1)$ & $38.3(6.9)$ & 0.65 \\
\hline Preintervention waist circumference, $\mathrm{cm}$ & $112.8(15.1)$ & $110.5(15.2)$ & 0.41 \\
\hline \multicolumn{4}{|l|}{ Plasma lipids, mean (SD) } \\
\hline Total cholesterol, mg/dl & $151.6(31.5)$ & $140.8(29.0)$ & 0.05 \\
\hline LDL cholesterol, mg/dl & $93.1(26.2)$ & $84.4(23.2)$ & 0.06 \\
\hline HDL cholesterol, mg/dl & 36.7 (9.9) & $35.5(10.8)$ & 0.54 \\
\hline Triglycerides, mg/dl & $108.9(40.3)$ & $104.7(33.0)$ & 0.54 \\
\hline \multicolumn{4}{|l|}{ BP, mean (SD) } \\
\hline Systolic BP, mmHg & $122.8(14.5)$ & $121.5(14.0)$ & 0.60 \\
\hline Diastolic BP, mmHg & $74(9.3)$ & $75.4(7.9)$ & 0.35 \\
\hline \multicolumn{4}{|l|}{ Medication use, no. (\%) } \\
\hline $\mathrm{BP}$ & $17(25.8)$ & $12(19.3)$ & 0.49 \\
\hline Cholesterol & $6(9.1)$ & $5(8.1)$ & 0.85 \\
\hline Antidepressant & $30(45.4)$ & $26(41.9)$ & 0.80 \\
\hline Hypothyroidism & $12(18.2)$ & $13(20.9)$ & 0.74 \\
\hline
\end{tabular}

Data show mean \pm SD of all subjects. SI conversion factors: to convert total cholesterol and HDL cholesterol values from $\mathrm{mg} / \mathrm{dl}$ to $\mathrm{mmol} / \mathrm{l}$, multiply by 0.0259 ; triglyceride values from $\mathrm{mg} / \mathrm{d}$ to $\mathrm{mmol} / \mathrm{l}$, by 0.0113 ; glucose values from $\mathrm{mg} / \mathrm{dl}$ to $\mathrm{mmol} / \mathrm{l}$, by 0.0555 ; and insulin values from $\mu \mathrm{lU} / \mathrm{ml}$ to $\mathrm{pmol} / \mathrm{l}$, by 6.945 .

structured exercise intervention on $\mathrm{S}_{\mathrm{I}}$, cardiorespiratory fitness, body composition, weight, and other cardiometabolic risk factors following RYGB surgery.

\section{Results}

Study participants. The flow of participants through the study is depicted in Figure 1. Forty-eight participants were randomized at the East Carolina University site $(n=24$, exercise program $[\mathrm{EX}] ; n=24$, health education control $[\mathrm{CON}])$ and 80 at the University of Pittsburgh site $(n=42$, $\mathrm{EX} ; n=38, \mathrm{CON}$ ). The average time from the date of RYGB surgery to randomization into study groups was $79 \pm 25$ days. Retention rates for the interventions were $95 \%$ for the CON group and $91 \%$ for the EX group $(P=0.49)$. There were no serious adverse events in either study group, nor were there differences in reported adverse events between groups. In the intention-to-treat (ITT) analysis, the EX group performed an average $( \pm$ SEM) of $147 \pm 15 \mathrm{~min} / \mathrm{wk}$ of exercise, measured over the final 12 weeks of the intervention. Forty-four out of sixty-six EX participants performed at least $120 \mathrm{~min} / \mathrm{wk}$ (average:

improve glucose effectiveness $\left(\mathrm{S}_{\mathrm{C}}\right)$ in healthy individuals following a 12-week exercise program (17). Exercise increases oxidative enzyme activity in skeletal muscle and induces related biochemical and morphological changes that would seem to confer a metabolic basis for improved $\mathrm{S}_{\mathrm{I}}(11,18)$.

Exercise in combination with caloric restriction can produce an additive reduction in body weight (10) and may be beneficial for weight-loss maintenance (19). However, it is not known whether exercise following bariatric surgery provides additional improvements in cardiometabolic risk factors, including $\mathrm{S}_{\mathrm{I}}$. Indeed, only one study to date has assessed whether aerobic exercise is even a feasible therapeutic option in this patient population (20). Case-controlled studies indicate that bariatric surgery patients have lower levels of physical activity than weight-matched nonsurgery controls (21-23). A number of retrospective cohort studies report a positive association between physical activity and postsurgery weight loss (24-28). However, these studies are limited by their observational nature, inclusion of only patients who return for follow-up, and subjectively measured physical activity by self-report questionnaires (29).

To date, there have been no randomized, controlled trials that have examined the effects of an exercise intervention on $S_{I}$ and other cardiometabolic risk factors following bariatric surgery. To address this paucity in the literature, we conducted a randomized, controlled trial to examine the effects of a 6-month semi-
$185 \pm 18 \mathrm{~min} /$ wk) of exercise and were thus included in the perprotocol (PP) analyses. For the $22 \mathrm{EX}$ participants who were not included in the PP analysis, 5 did not complete the intervention due to time-commitment issues and 1 was lost to follow-up. The other 16 participants who did complete postintervention testing averaged only $55.7 \mathrm{~min} /$ wk of exercise during the final 3 months of the intervention. There were a number of reasons for noncompliance to the exercise intervention. Five participants reported health problems (knee pain, hernia, rotator cuff surgery) not related to the study that prevented $120 \mathrm{~min} /$ wk of exercise. Two participants lived more than 35 miles from the exercise facility, which made compliance difficult. Nine participants did not have an apparent reason for noncompliance. Six participants in the CON group reported a physically active lifestyle (exercising $>1$ day/wk), so 56 out of $62 \mathrm{CON}$ participants randomized were included in the PP analysis.

Baseline characteristics of the study groups are shown in Table 1. Of the 128 participants randomized, 15 were male and 113 were female, and 22 were African-American and 106 were of mixed European descent. There were no race or sex imbalances between groups. There were no differences in demographics or characteristics contributing to outcomes between study groups. Total and LDL cholesterol tended to be higher in the EX group than in the CON group. The groups had similar body weight presurgery and preinterventions. Both groups also reported similar 


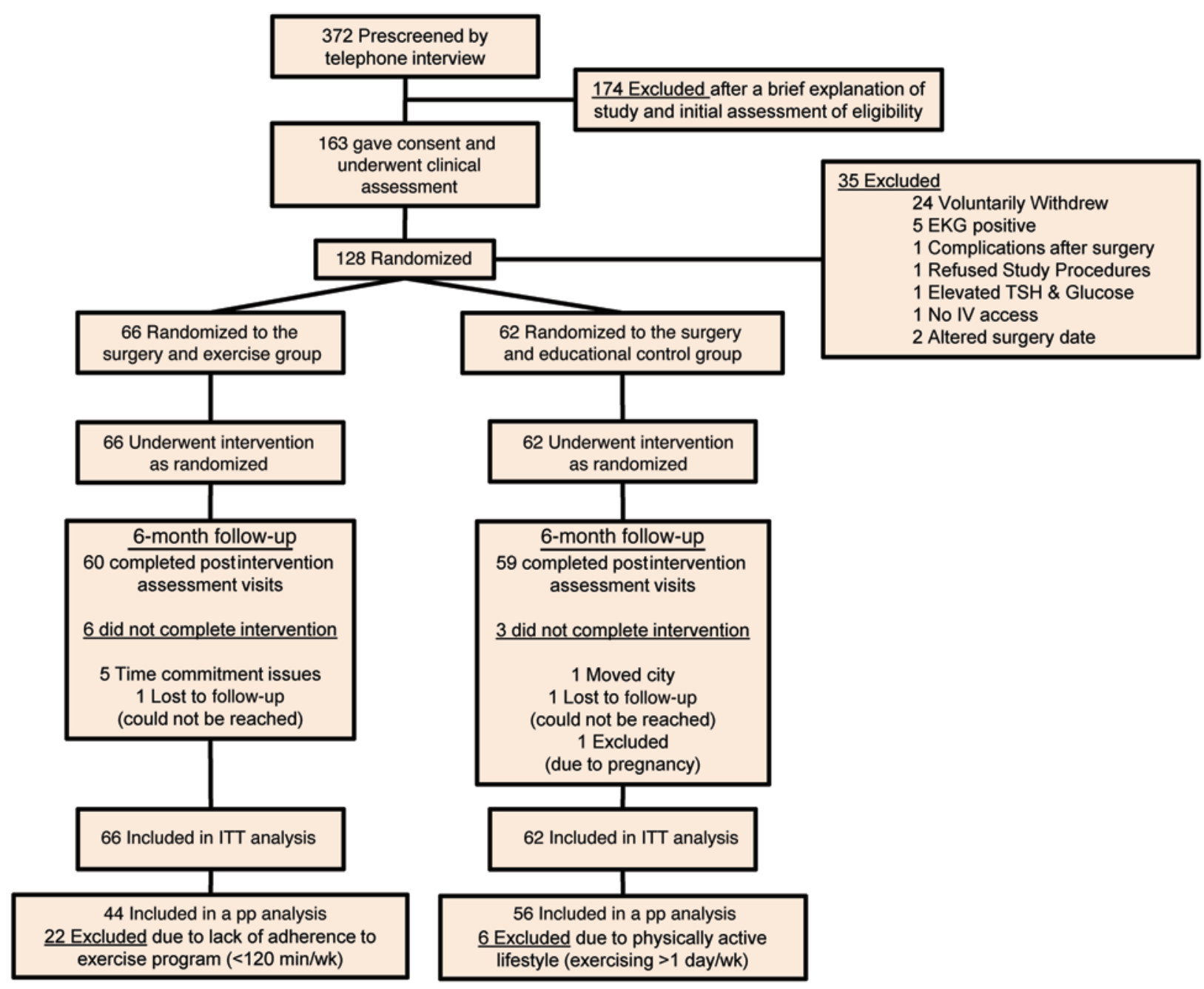

Figure 1. Flow of participant recruitment, screening, and assessment.

medication use at baseline. There were no group differences in medication use following intervention.

Intravenous glucose tolerance test. Preintervention $\mathrm{S}_{\mathrm{I}}$ measurements for both groups were similar, but substantially lower than those observed for normal-weight subjects of a similar age (3). Following the interventions, there was a significant time effect $(P<0.001)$, with both groups showing improved $\mathrm{S}_{\mathrm{I}}$ (Table 2 and Figure 2). In the ITT analysis, $S_{I}$ tended to improve to a greater degree for the EX group compared with the CON group, but this was not statistically different between groups (Figure 2: EX vs. CON; +2.24 vs. $\left.+1.64 \mathrm{~min}^{-1} / \mu \mathrm{U} / \mathrm{ml}, P=0.18\right)$. Next, in a $\mathrm{PP}$ analysis, we examined the effects of exercise for individuals who met the a priori-defined intervention protocol criteria (Table 3 and Figure 2). In this analysis, those subjects who performed more than $120 \mathrm{~min} / \mathrm{wk}$ of exercise in the final 3 months of the intervention had significantly greater improvements in $S_{I}$ compared with those observed for RYGB surgery-induced weight loss alone (Figure 2: EX vs. CON; +2.69 vs. +1.57, $\left.\mathrm{min}^{-1} / \mu \mathrm{U} / \mathrm{ml}, P=0.019\right)$. The plasma insulin and glucose excursions during the intravenous glucose tolerance test (IVGTT) for the PP analysis are presented in Figure 3.
$\mathrm{S}_{\mathrm{G}}$ refers to the ability of glucose per se to stimulate glucose uptake and is an important component of glucose metabolism. $\mathrm{S}_{\mathrm{G}}$ was similar at baseline (EX vs. CON; 0.015 vs. $0.017 \mathrm{~min}^{-1}$ ). ITT analysis revealed that there was a main effect of surgery (time) for $\mathrm{S}_{\mathrm{G}}$ (Table 2: $\left.P<0.001\right)$ and that there was a greater improvement observed in the EX group (Figure 4: EX vs. CON; +0.0063 vs. $+0.0023 \mathrm{~min}^{-1}, P=0.009$ ). The exercise effect on $\mathrm{S}_{\mathrm{G}}$ was also robust in the PP analysis (Figure 4: EX vs. CON; +0.0071 vs. $\left.+0.0023 \mathrm{~min}^{-1}, P=0.011\right)$. Thus, regular exercise following RYGB surgery elicits a powerful effect on the ability of glucose per se to stimulate glucose uptake. Acute insulin response (AIRg) and disposition index $\left(\mathrm{D}_{\mathrm{I}}\right)$ improved over time, but there was no additional improvement with exercise (Tables 2 and 3).

Weight, body composition, and waist circumference. Both study groups exhibited similar mass, BMI, waist circumference, and total and depot-specific fat mass at baseline and similar reductions when the data were analyzed with ITT and PP approaches (Tables 2 and 3). There was no difference in the loss of subcutaneous and visceral fat depots after the interventions. We did not observe a difference in proportionate weight loss between fat depots. 

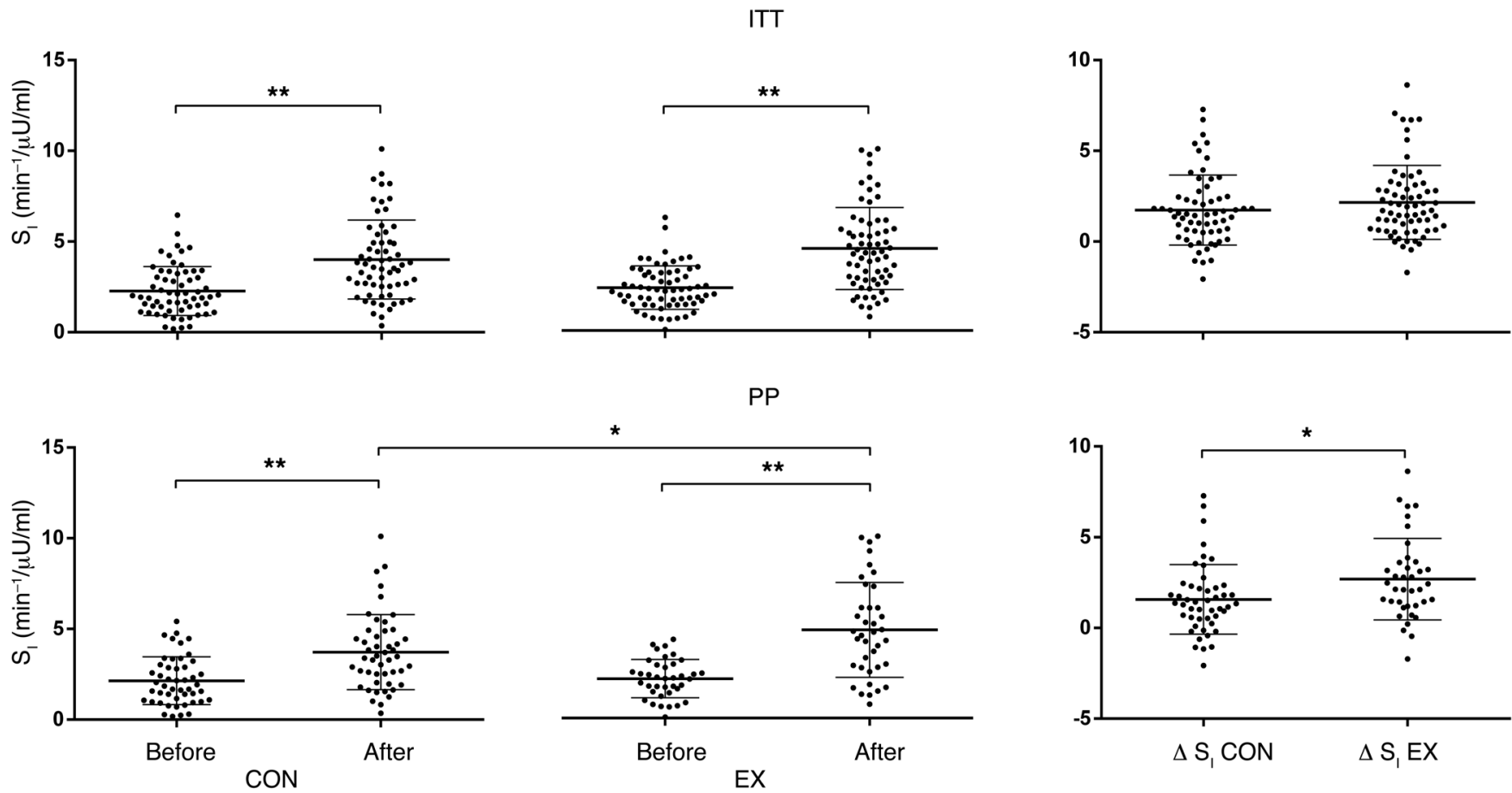

Figure 2. ITT and PP analysis for change in $\mathbf{S}_{1}$ in exercise and control groups. Data shown are mean \pm SD for all subjects, with MCMC MI for missing data in ITT analysis. For the PP analysis, only subjects who complied with intervention protocols and completed at month 6 were included. ${ }^{*} P<0.05 ;{ }^{* *} P<0.01$.

Other cardiometabolic risk factors. Compared with CON, EX significantly improved $\mathrm{VO}_{2}$ peak (Figure 5), an index of cardiorespiratory fitness and an effective predictor of future morbidity and mortality (30). Blood pressure (BP) (systolic and diastolic), cholesterol (total, LDL, and HDL), and triglycerides were reduced to a similar degree in the EX and CON groups (ITT, Table 2; PP, Table 3 ); there was no additional effect of exercise on these cardiometabolic risk factors.

\section{Discussion}

RYGB surgery is a highly effective treatment option for severe obesity, as it results in substantial weight loss and improved cardiometabolic risk profile, including $\mathrm{S}_{\mathrm{I}}$. Although exercise can also reduce cardiometabolic risk independently of weight, it is not known whether exercise can promote additional improvements in RYGB surgery patients concomitant with their robust weight loss. Our results indicate that moderate aerobic exercise elicits additional improvements in $\mathrm{S}_{\mathrm{I}}$ as well as $\mathrm{S}_{\mathrm{G}}$, i.e., the ability of glucose per se to facilitate glucose disposal, along with improved cardiorespiratory fitness concomitant with RYGB surgery-induced weight loss. These data advocate for the inclusion of an exercise program to optimize health benefits during active weight loss following RYGB surgery.

The improvement in glucose control in T2D patients following RYGB surgery may be due to energy restriction-induced improvements in hepatic $\mathrm{S}_{\mathrm{I}}$ and a modified postprandial gut hormone response in the days to weeks after the operation (7). Improved peripheral $\mathrm{S}_{\mathrm{I}}$, however, appears to be more strongly associated with longer-term weight loss $(3,7)$. Here, we recapitulate these findings and demonstrate that, despite substantial initial weight loss ( 20 kg), within 1 to 3 months after surgery, peripheral $S_{I}$ remains approximately $60 \%$ lower compared with that of metabolically healthy lean individuals of similar age and the same sex (31). Our data affirm that there is capacity for further improvements through lifestyle interventions, such as exercise.

Our PP analysis demonstrates that an exercise program of more than 120 min/wk effectively improved $S_{I}$ in RYGB surgery patients, by approximately $30 \%$ over a sedentary lifestyle. These data are the first, to our knowledge, to suggest that an exercise intervention was not only feasible, but was efficacious in improving $\mathrm{S}_{\mathrm{I}}$ in severely obese RYGB surgery patients. This observation is congruent with the finding that a 12 -week exercise program following RYGB and gastric banding improved glucose tolerance (20). In the current study, exercise-induced improvements in $S_{I}$ and $S_{G}$ cannot be accounted for by any additional effect of exercise on reduced body mass or adiposity. This does not, however, preclude the possibility that improvements in $\mathrm{S}_{\mathrm{I}}$ with exercise were related to changes in intramyocellular lipids, inflammation, or other factors that influence insulin action (32).

We observed a robust and statistically significant improvement in $S_{G}$ with exercise after surgery. These data indicate that an exercise program during RYGB surgery-induced weight loss may improve metabolic health by both insulin-dependent and -independent mechanisms (at basal insulin levels). $\mathrm{S}_{\mathrm{G}}$ reflects the capacity of glucose per se to enhance cellular glucose uptake and accounts for approximately $50 \%$ of glucose disposal following a meal $(33,34)$. The cellular mechanisms that mediate $S_{G}$ are unclear, but are thought to relate to glucose mass action (35). $S_{G}$ is an independent predictor of diabetes across race/ethnic groups and varying degrees of obesity and is reduced with impaired 
Table 2. ITT analysis of weight, body composition, cardiorespiratory fitness, and IVGTT variables

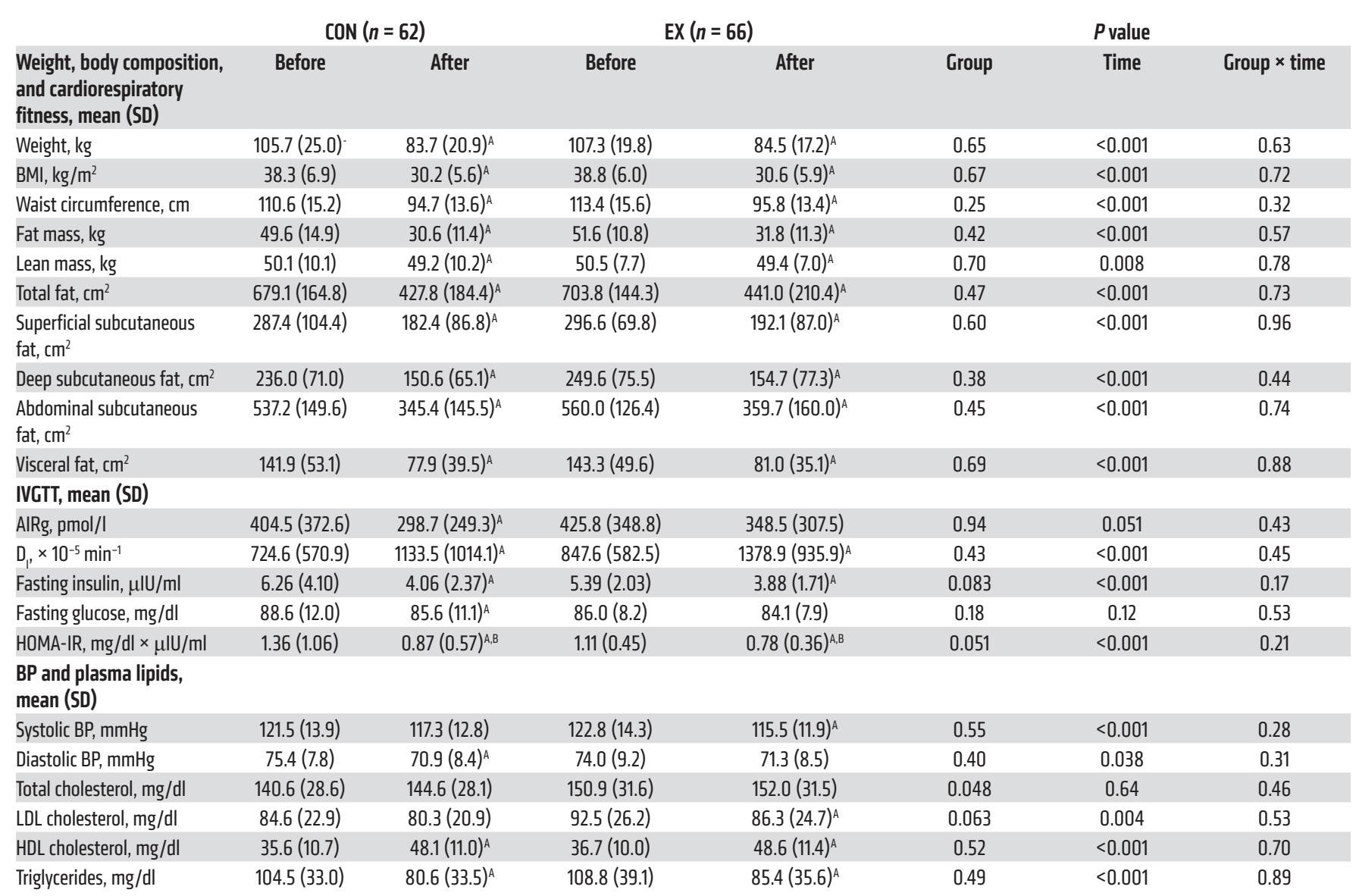

Data show mean \pm SD of all subjects, with MCMC MI for missing data., ${ }^{A}$ Within group, significant difference from before to after, with FDR $P<0.05$. ${ }^{B}$ Between groups, significant difference at before and after, with FDR $P<0.05$. FFM, fat-free mass; HOMA-IR, homeostasis model assessment of insulin resistance (fasting glucose $\times$ fasting insulin)/22.5. S conversion factors: to convert total cholesterol and HDL cholesterol values from mg/dl to $\mathrm{mmol} / \mathrm{l}$, multiply by 0.0259 ; triglyceride values from $\mathrm{mg} / \mathrm{dl}$ to $\mathrm{mmol} / \mathrm{l}$, by 0.0113 ; glucose values from $\mathrm{mg} / \mathrm{dl}$ to $\mathrm{mmol} / \mathrm{l}$, by 0.0555 ; and insulin values from $\mu \mathrm{lU} / \mathrm{ml}$ to $\mathrm{pmol} / \mathrm{l}$, by 6.945 .

glucose tolerance, diabetes, and aging (36). $\mathrm{S}_{\mathrm{G}}$ is not affected by calorie restriction (37) or vertical banded gastroplasty (38). However, Nishida et al. found that $S_{G}$ improved in healthy individuals following a 12-week exercise program (17). These studies support our contention that exercise-mediated improvements in $\mathrm{S}_{\mathrm{G}}$ in RYGB patients represent a physiologically and potentially clinically important finding, although the mechanism or mechanisms by which this occur are not clearly evident.

The physical activity habits of bariatric surgery patients have not been clearly described. Case-control studies report significantly lower levels of physical activity than weight-matched nonsurgery controls (21-23). Indeed, it is not clear whether an exercise training intervention is even a feasible therapeutic option in this unique patient population. Here, we show that $91 \%$ of patients randomized to the exercise group completed the intervention. We also used a PP approach using only data from subjects who adhered to the a priori-defined exercise prescription and intervention, which is within recommended guidelines. The exercise program (>120 $\mathrm{min} / \mathrm{wk}$ ) was adhered to by two-thirds of the patients randomized to the exercise group, who exercised an average of $185 \mathrm{~min} / \mathrm{wk}$. These data indicate that exercise training is a feasible treatment option for this patient population. Of the 22 participants who did not strictly adhere to the intervention, 13 reported reasons for noncompliance, while 9 did not. It has been well documented that bariatric surgery patients have psychosocial barriers to exercise, including low self efficacy and exercise motivation, and social stigma $(39,40)$. The aim of this study was not to determine psychosocial barriers to exercise, and while we didn't measure these factors, it is likely that they contributed to noncompliance in these 9 participants.

Exercise following RYGB surgery also significantly improved cardiorespiratory fitness ( $\mathrm{VO}_{2}$ peak). This finding is similar to other reports of improvements in fitness with exercise in post-bariatric surgery patients (20) and is clinically important, as cardiorespiratory fitness is inversely correlated with BMI (41) and improved cardiorespiratory fitness is associated with a reduced risk of allcause mortality (42). Therefore, not only is an aerobic exercise training program feasible in this population, it is also effective at 

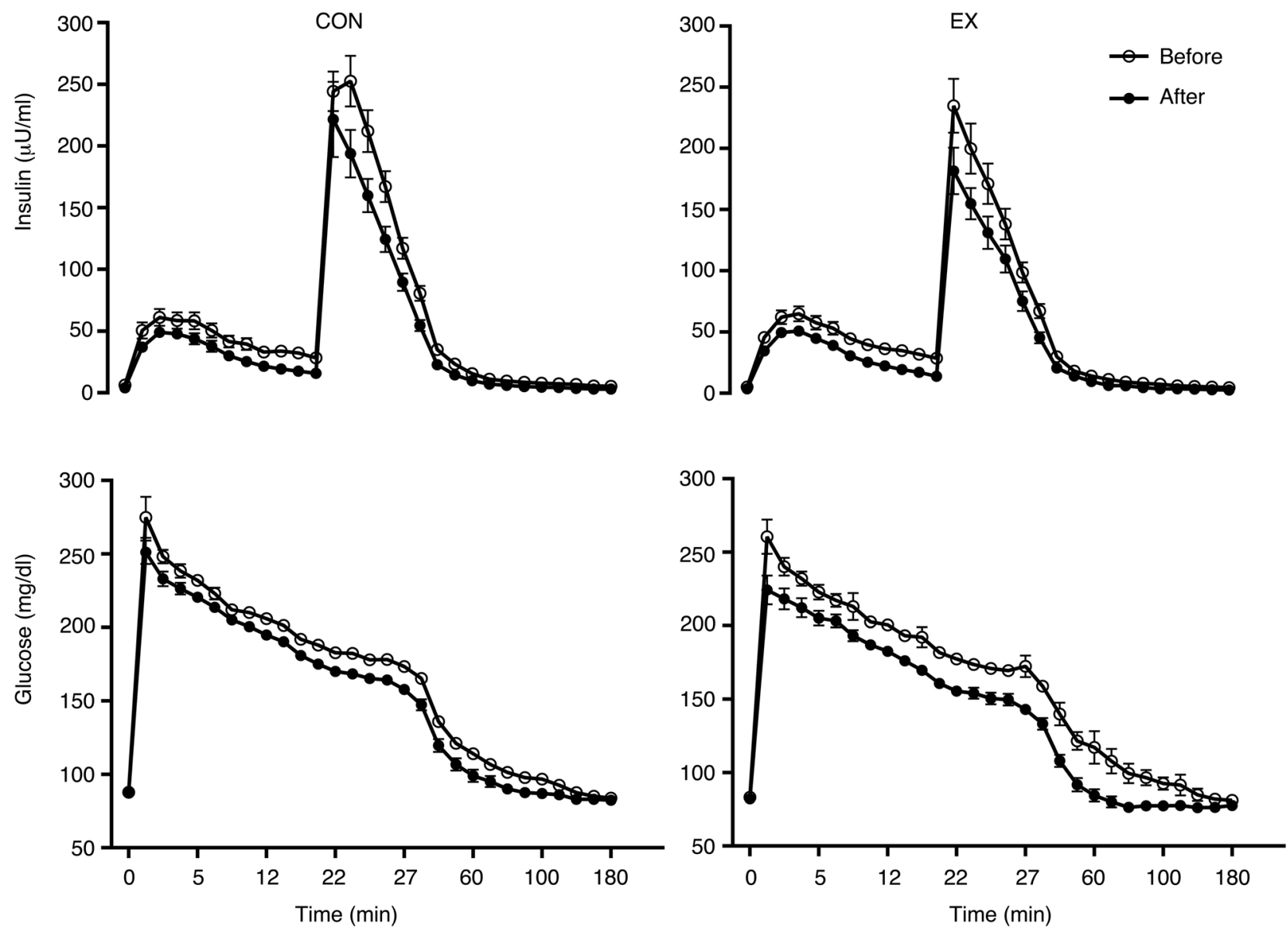

Figure 3. Plasma glucose and insulin concentrations during the 3-hour IVGTT in exercise and control groups. Data shown are mean \pm SEM. For the PP analysis, only subjects who complied with and completed the intervention protocols were included.

improving cardiorespiratory fitness, a result that directly counters the perception that severely obese individuals cannot respond to lifestyle interventions. Our data highlight the need for additional randomized controlled exercise trials to better understand the implications of components of exercise prescription (dose, duration, intensity) on long-term weight loss maintenance and health in RYGB surgery patients.

Increased physical activity after bariatric surgery has been reported to provide additional weight loss (43). However, most of these studies were nonrandomized, retrospective, and observational in nature and measured physical activity by questionnaire and self report (43), methods that may lead to overestimation of exercise participation in obese subjects $(44,45)$, including those who have undergone bariatric surgery (29). Our observations using a semi-supervised exercise intervention are in line with those of Shah et al., who showed that a high-volume exercise prescription following bariatric surgery had no impact on body weight and waist circumference when patients were compared with a control group (20). We extend these observations by demonstrating that abdominal adipose depots, measured by computed tomography (CT), are not uniquely influenced by a 6 -month exercise training intervention in RYGB surgery patients.

Strengths and limitations. A particular strength of this study is that it is, we believe, the first randomized controlled trial in a rea- sonably large sample of RYGB surgery patients to examine the efficacy of a semi-supervised exercise program on $\mathrm{S}_{\mathrm{r}}$. Although the adherence was high in our study, we did not determine the feasibility or the efficacy of exercise in a broader group of patients or across different bariatric surgery procedures. Therefore, we cannot generalize the potential benefits of exercise after bariatric surgery. Additional studies are needed to determine the optimal dose and modality of exercise and whether exerciseinduced benefits extend to other bariatric surgery procedures. The semi-supervised nature of the intervention indicates that it is clinically practical and the prescription performed is feasible and well tolerated by RYGB surgery patients, which has important implications for refining future physical activity recommendations. Our study had some additional limitations. Participants were mostly younger to middle-age women, and although randomized groups were stratified by sex, it is difficult to determine sex-specific responses. Nutritional intake was not controlled or monitored and may represent an important factor that contributes to affecting outcome measures, including $\mathrm{S}_{\mathrm{I}}$, weight loss, and other cardiometabolic risk factors.

Conclusions. RYGB surgery patients who regularly perform a modest amount $(>120 \mathrm{~min} / \mathrm{wk}$ ) of exercise achieve significant improvements in $\mathrm{S}_{\mathrm{I}}$ and $\mathrm{S}_{\mathrm{G}}$ beyond those derived from RYGB surgery-induced weight loss alone. Thus, exercise can be a useful 


\section{Table 3. PP analysis of weight, body composition, cardiorespiratory fitness, and IVGTT variables}

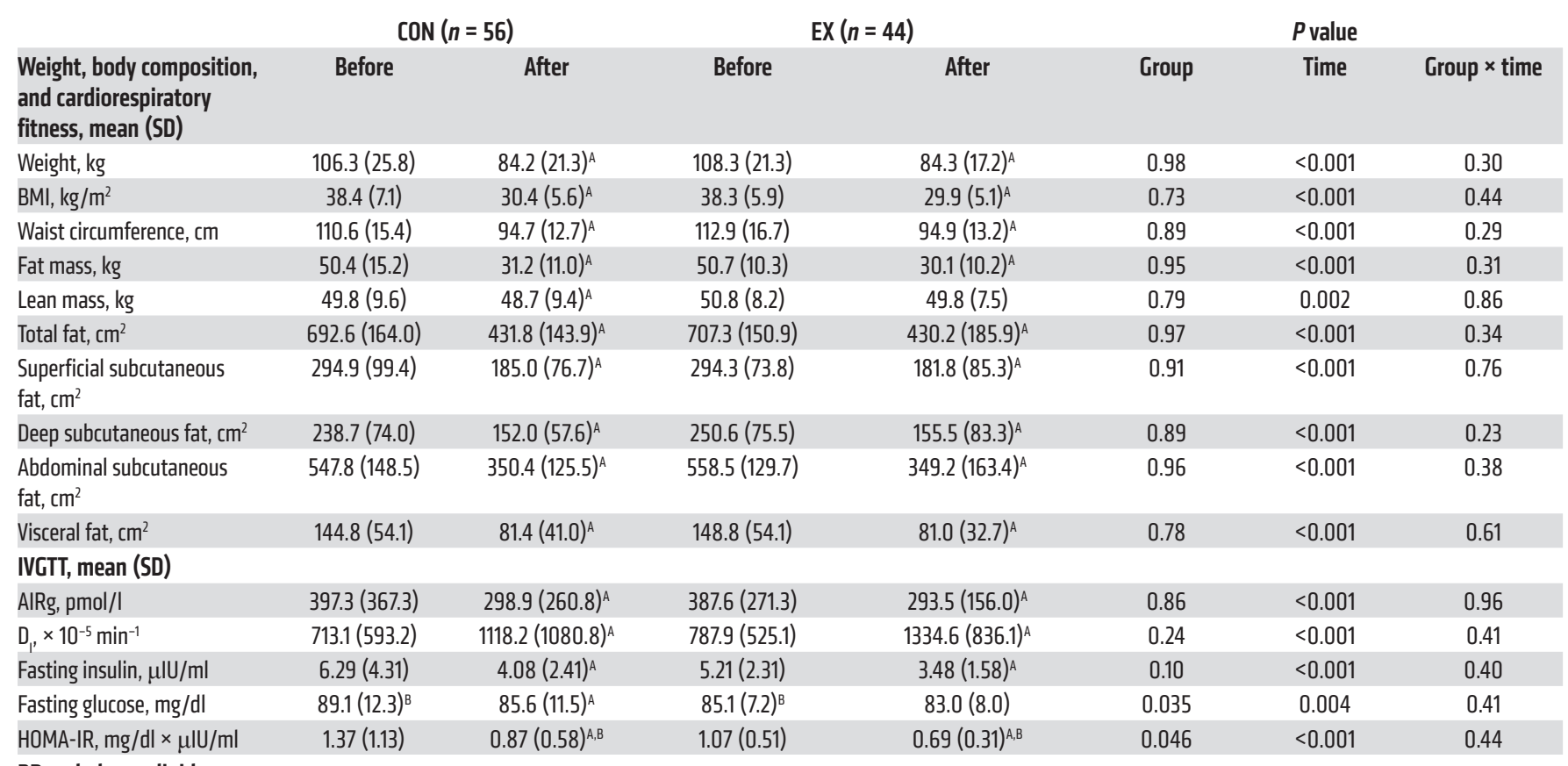

\section{$\mathrm{BP}$ and plasma lipids, mean}

(SD)

\begin{tabular}{lccccccc} 
Systolic BP, mmHg & $122.4(14.0)$ & $117.0(13.0)^{\mathrm{A}}$ & $121.7(13.7)$ & $115.4(12.1)^{\mathrm{A}}$ & 0.51 & $<0.001$ & 0.78 \\
Diastolic BP, mmHg & $75.9(7.9)$ & $70.8(8.6)^{\mathrm{A}}$ & $73.1(9.4)$ & $70.8(8.1)$ & 0.20 & $<0.001$ & 0.18 \\
Total cholesterol, mg/dl & $142.5(28.7)$ & $146.5(27.5)$ & $149.6(31.7)$ & $149.5(32.0)$ & 0.29 & 0.34 & 0.32 \\
LDL cholesterol, mg/dl & $85.6(23.2)$ & $81.7(20.3)$ & $92.7(25.3)$ & $86.1(24.1)$ & 0.15 & 0.003 & 0.44 \\
HDL cholesterol, mg/dl & $36.0(10.9)$ & $48.7(10.9)^{\mathrm{A}}$ & $35.7(9.9)$ & $47.4(11.4)^{\mathrm{A}}$ & 0.89 & $<0.001$ \\
Triglycerides, mg/dl & $105.3(33.3)$ & $80.1(33.9)^{\mathrm{A}}$ & $106.3(40.7)$ & $80.5(31.5)^{\mathrm{A}}$ & 0.94 & $<0.001$ & 0.72 \\
\hline
\end{tabular}

Data are mean \pm SD for all subjects. For the PP analysis, only subjects who complied with intervention protocols and completed the intervention were included., ${ }^{A}$ Within group, significant difference from before to after, with FDR $P<0.05$. ${ }^{B}$ Between groups, significant difference at before and after, with FDR $P<0.05$. HOMA-IR: (fasting glucose $\times$ fasting insulin)/22.5. S, conversion factors: to convert total cholesterol and HDL cholesterol values from mg/dl to $\mathrm{mmol} / \mathrm{l}$, multiply by 0.0259 ; triglyceride values from $\mathrm{mg} / \mathrm{dl}$ to $\mathrm{mmol} / \mathrm{l}$, by 0.0113 ; glucose values from $\mathrm{mg} / \mathrm{dl}$ to $\mathrm{mmol} / \mathrm{l}$, by 0.0555 ; and insulin values from $\mu \mathrm{lU} / \mathrm{ml}$ to $\mathrm{pmol} / \mathrm{l}$, by 6.945 .

adjunct therapy for RYGB surgery patients to promote additional improvements in cardiometabolic risk and physical fitness.

\section{Methods}

Patient recruitment. RYGB surgery patients (1 to 3 months after surgery) were recruited from the University of Pittsburgh Medical Center and East Carolina University bariatric surgery centers in Pittsburgh, Pennsylvania, USA, and Greenville, North Carolina, USA. The study setting was an academic clinical translational research center. Recruitment commenced in September 2008, the last participant was randomized in March 2012, and data were available for analysis in October 2012.

Inclusion/exclusion criteria. Male and female participants were eligible if they were between 21 and 60 years, had a BMI below $55 \mathrm{~kg} / \mathrm{m}^{2}$, and underwent RYGB surgery 1 to 3 months previously. Race/ethnicity was self reported. Participants were required to walk without assistance. If on hormone replacement therapy, participants remained on the same dose throughout the study. Participants were excluded if they had a diagnosis of diabetes, hypertension, anemia, hypothyroidism, elevated liver enzymes, current malignancy or history of cancer within past 5 years, or stent placement within the past 3 years. Participants were also excluded if there was a history of myocardial infarction, angioplasty, angina, liver disease, or neuromuscular disease. Medication exclusions included the following: anticoagulation therapy, steroids or other drugs that would alter metabolism, glucose homeostasis, or medications that would confound study results. Participants were also excluded if they reported being physically active, defined as participating in planned exercise ( $>30 \mathrm{~min}$ in duration) more than 1 day a week, as determined by self report.

Randomization. A permuted-blocks approach was used, with subjects stratified by gender. Blocks of random sizes of 4 and/or greater were used to achieve the goal sample size in each group between both study sites. The study clinical coordinator at Pittsburgh conducted randomization for both sites. The study was single blind, with assessors for all outcomes blinded to participant group assignment.

Intervention groups. Participants (1 to 3 months following surgery) were randomized to 6-month semi-supervised EX or CON intervention. Participants were required to participate in 3 to 5 exercise sessions per week, with at least 1 directly supervised session per week 

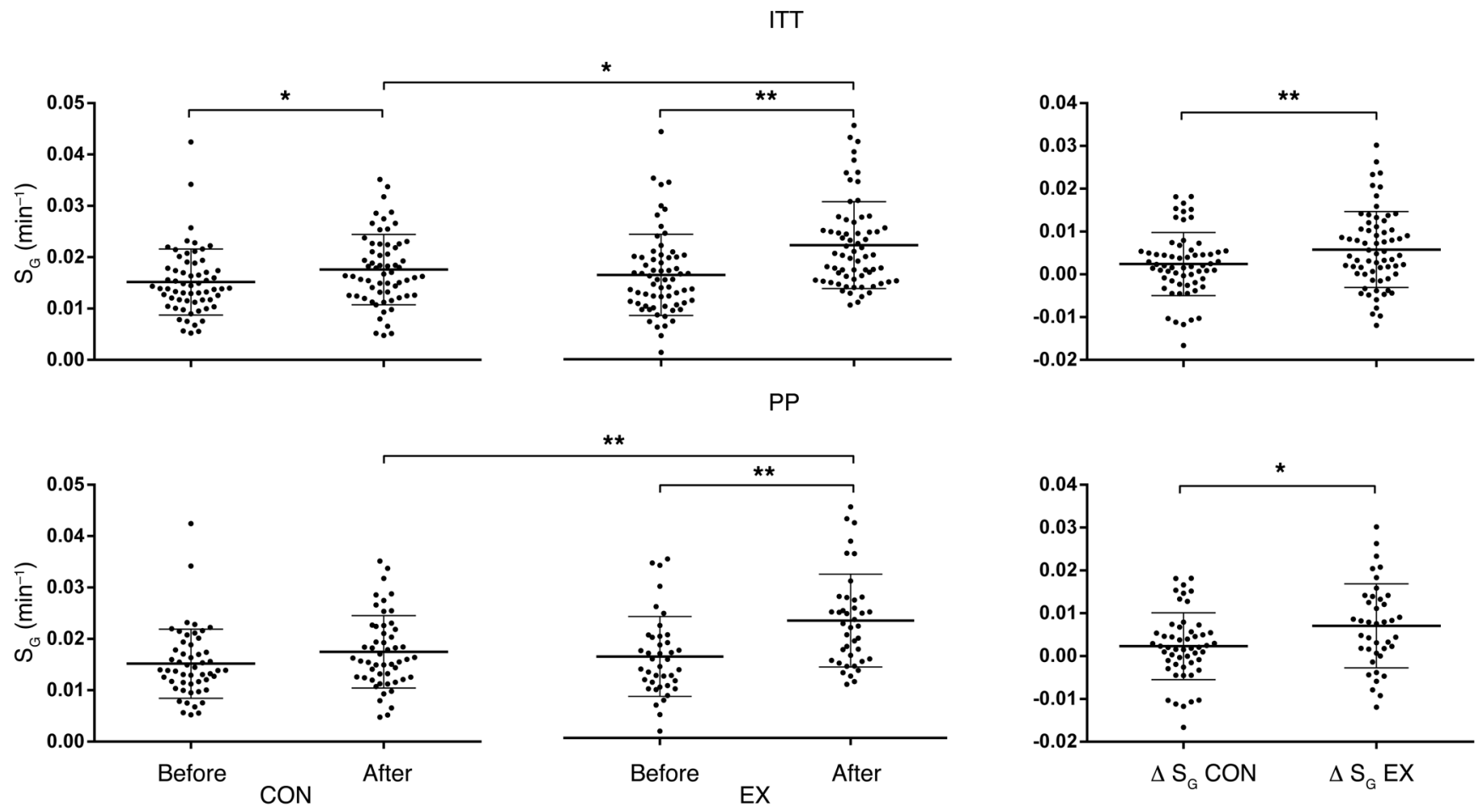

Figure 4. ITT and PP analysis for change in $\mathbf{S}_{\mathbf{c}}$ in exercise and control groups. Data shown are mean \pm SD for all subjects, with MCMC MI for missing data in ITT analysis. For the PP analysis, only subjects who complied with intervention protocols and completed at month 6 were included. ${ }^{*} P<0.05$; ${ }^{* *} P<0.01$.

to assure that target exercise intensity and duration were achieved and to discuss and document progress. Participants used a heart-rate monitor and recorded detailed logs of their exercise sessions, including type of exercise, duration, and average heart rate. Intervention compliance of the EX participants was monitored and encouraged weekly by a trained exercise physiologist.

For the first 4 weeks, participants exercised for as long as feasible ( 10-15 $\mathrm{min}$ ) per session at an intensity level of $60 \%$ to $70 \%$ of their maximal heart rate. Participants progressed over 3 months to a minimum of $120 \mathrm{~min} /$ wk of exercise, which was maintained for the final 3 months of the program. Exercise consisted of stationary cycling or treadmill walking at home if this equipment was available or walking or cycling outdoors for a similar duration. Due to potential functional limitations to exercise caused by obesity, participants were encouraged to accumulate the minimal level of exercise per day (30 $\mathrm{min} / \mathrm{d})$ in either an intermittent or continuous manner.

Health education control group participants were asked to attend 6 health education sessions. The sessions were held once per month and included lectures, discussions, and demonstrations providing up-to-date information on topics such as medication use, nutrition, and upper-body stretching. The participants in the EX group also received the same health education sessions, including advice on nutrition (6 sessions, 1 every month). CON participants also reported physical activity habits at the health education sessions.

Strategies to maintain adherence and reduce participant dropout included regular support from study personnel and remuneration of $\$ 200$ following baseline testing and $\$ 200$ for completion of postintervention testing.
Primary and secondary outcome measures. Study measurements were made over separate clinic visits before and after the 6-month interventions. A 3-hour insulin-modified IVGTT was performed in the morning hours after a 12-hour fast to determine insulin action parameters based on the Bergman minimal model calculations (46). A $50 \%$ dextrose bolus $(0.3 \mathrm{~g} / \mathrm{kg}$ body mass $)$ was administered after fasting samples were collected, and insulin ( $0.025 \mathrm{U} / \mathrm{kg}$ body mass) was then injected at minute 20. Blood samples were collected in EDTA tubes at minutes 2, 3, 4, 5, 6, 8, 10, 12, 14, 16, 19, 22, 23, 24, 25, $27,30,40,50,60,70,80,90,100,120,140,160$, and 180. Plasma samples were stored at $-80^{\circ} \mathrm{C}$ until later analyses. Plasma insulin was determined by enzyme immunoassay (Access Immunoassay System, Beckman Coulter) and glucose by the oxidation reaction (Sycron DxC600i, Beckman Coulter). $\mathrm{S}_{\mathrm{I}}, \mathrm{S}_{\mathrm{G}}, \mathrm{D}_{\mathrm{I}}$, and AIRg were calculated with MINMOD millennium software (version 5.10, 2002) (47).

Secondary measures included fat and lean mass determined by dual-energy x-ray absorptiometry (DXA) using a GE Lunar (GE Healthcare). Abdominal visceral and subcutaneous adipose tissue area were quantified by CT using SliceOmatic image analysis software (TomoVision) (48).

Cardiorespiratory fitness $\left(\mathrm{VO}_{2}\right.$ peak) was measured by indirect calorimetry during a 5- to 12-minute graded exercise test on a cycle ergometer (Lode) (49). Twelve-lead ECG recordings were monitored by the study physician and interpreted for contraindications to exercise. Body weight, BP, and plasma lipids and hepatic enzymes were measured by standard clinical protocols.

Power and sample size calculations. Power analyses were conducted a priori based on data from pilot studies at our 2 centers and indicated that a sample size of 63 subjects per group would provide $80 \%$ power 

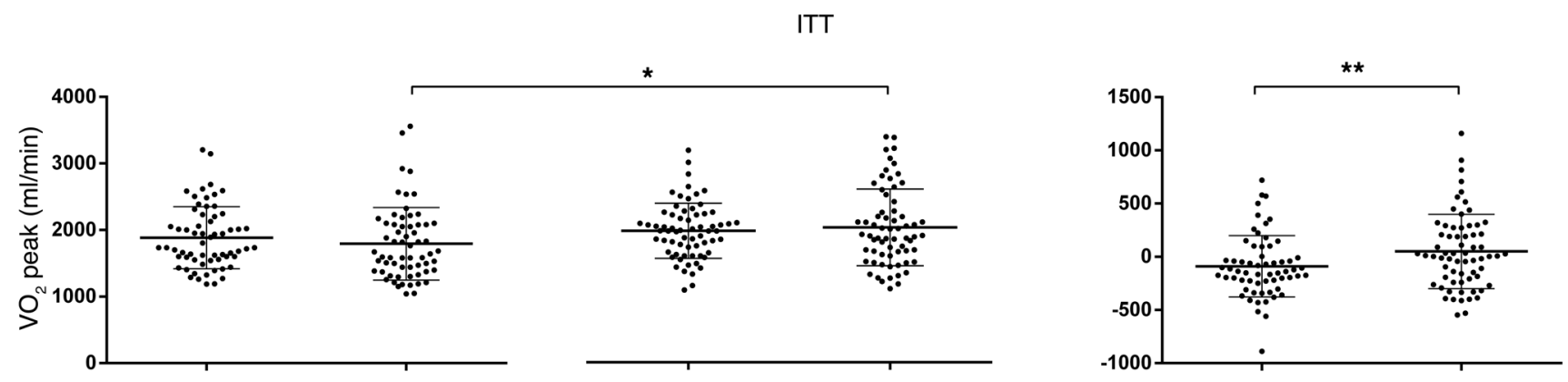

PP
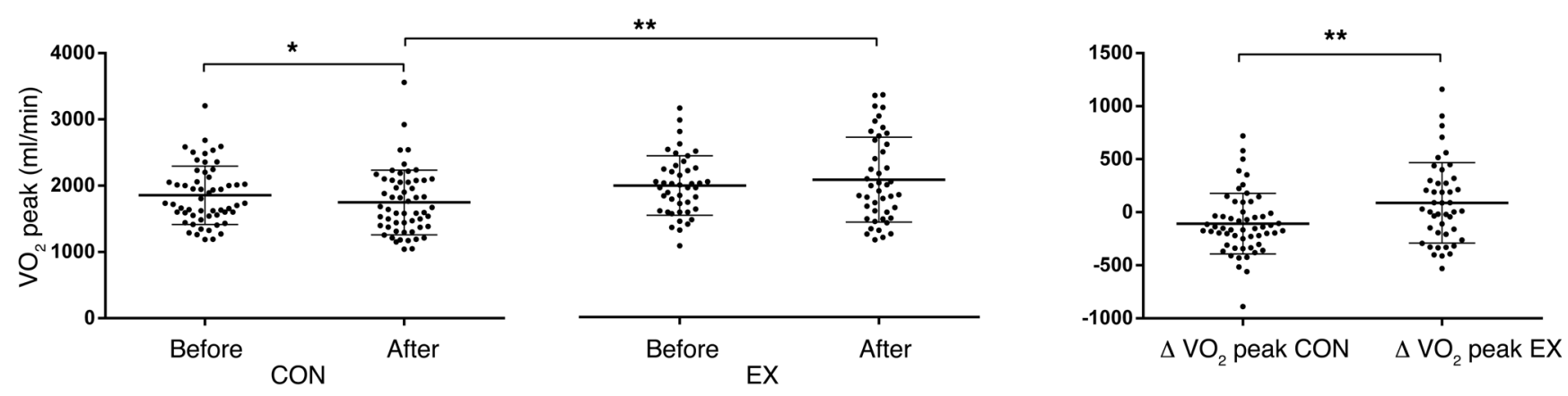

Figure 5. ITT and PP analysis for change in cardiorespiratory fitness in exercise and control groups. Data shown are mean \pm SD for all subjects, with MCMC MI for missing data in ITT analysis. For the PP analysis, only subjects who complied with intervention protocols and completed at month 6 were included. ${ }^{*} P<0.05 ;{ }^{* *} P<0.01$.

to detect a medium effect size (0.55) for statistically significant differences in $\mathrm{S}_{\mathrm{I}}$ and visceral abdominal fat.

Statistics. Group differences in baseline characteristics were determined using 2-sample Student's $t$ test (2 tailed) or $\chi^{2}$ or Fisher exact tests. The primary analyses were as follows: (a) ITT approach, in which all 128 randomized participants were assumed to adhere to treatment assignments and complete follow-up assessment and (b) a PP approach. Only EX participants who performed at least $120 \mathrm{~min} / \mathrm{wk}$ of exercise on average during the last 3 months of the intervention and CON participants who maintained a physically inactive lifestyle (exercising an average of $<1$ day/wk during the last 3 months of the intervention) were included in the PP analysis.

The multiple imputation (MI) method with Markov chain Monte Carlo (MCMC) algorithms was used to estimate missing data. Before MI, any variables with high skew were log- or square-root transformed to achieve a normal distribution. These variables were antitransformed after MI procedure. The general linear mixed model with repeated measures (PROC MIXED) was performed to detect group and time effects on the outcome variables. Group, time, and group multiplied by time were treated as fixed effects and subjects nested within each group as random effect. Age, sex, and race were covariates. We applied PROC MIANALYSIS to combine the statistical results generated from PROC MIXED based on each imputation data. $P$ values for post-hoc tests were adjusted by false discovery rate (FDR). A $P$ value of less than 0.05 was considered significant. Analyses were performed using SAS version 9.1 (SAS Institute Inc.).

Study approval. The study protocol was approved by the University of Pittsburgh and East Carolina University Institutional Review Boards and was conducted in accordance with the Declaration of Helsinki. All participants provided written informed consent prior to their participation in the study. The study was registered at www.clinicaltrials.gov (trial ID NCT00692367).

\section{Acknowledgments}

The authors would like to thank the following individuals: Steven R. Smith, for his valuable input regarding data analysis; Marisa E. Desimone, for clinical support; Steven Anthony, for administering the exercise and nutrition interventions at the University of Pittsburgh; Alex Despines, for CT scan analysis; and the staff at the Clinical Translational Research Center, University of Pittsburgh. J.M. Jakicic and G.M. Eid received grants from the NIH. This study was funded by a grant from the NIH/National Institute of Diabetes and Digestive and Kidney Diseases (R01 DK078192) and an NIH/National Center for Research Resources/Clinical and Translational Science Award (UL1 RR024153).

Address correspondence to: Bret H. Goodpaster, Translational Research Institute for Metabolism and Diabetes, Florida Hospital - Sanford-Burnham Medical Research Institute, 301 East Princeton Street, Orlando, Florida 32804, USA. Phone: 407.303.1305; E-mail: Bret.Goodpaster@FLHosp.org.

Paul M. Coen and Bret H. Goodpaster's present address is: Translational Research Institute for Metabolism and Diabetes, Florida Hospital, Orlando, Florida, USA.

George M. Eid's present address is: Department of Surgery, Allegheny Health Network, Pittsburgh, Pennsylvania, USA. 
1. Schauer PR, et al. Effect of laparoscopic Rouxen $Y$ gastric bypass on type 2 diabetes mellitus. Ann Surg. 2003;238(4):467-484.

2. Buchwald $\mathrm{H}$, et al. Weight and type 2 diabetes after bariatric surgery: systematic review and metaanalysis. Am J Med.2009;122(3):248-256.e5.

3. Reed MA, et al. Roux-en-Y gastric bypass corrects hyperinsulinemia implications for the remission of type 2 diabetes. JClin Endocrinol Metab. 2011;96(8):2525-2531.

4. Dunn JP, et al. Hepatic and peripheral insulin sensitivity and diabetes remission at 1 month after Roux-en-Y gastric bypass surgery in patients randomized to omentectomy. Diabetes Care. 2012;35(1):137-142.

5. Camastra S, et al. Early and longer term effects of gastric bypass surgery on tissue-specific insulin sensitivity and $\beta$ cell function in morbidly obese patients with and without type 2 diabetes. Diabetologia. 2011;54(8):2093-2102.

6. Lima MM, et al. Acute effect of roux-en-y gastric bypass on whole-body insulin sensitivity: a study with the euglycemic-hyperinsulinemic clamp. JClin Endocrinol Metab. 2010;95(8):3871-3875.

7. Dirksen C, et al. Mechanisms of improved glycaemic control after Roux-en-Y gastric bypass. Diabetologia. 2012;55(7):1890-1901.

8. Bradley D, et al. Gastric bypass and banding equally improve insulin sensitivity and $\beta$ cell function. J Clin Invest. 2012;122(12):4667-4674.

9. Lim EL, Hollingsworth KG, Aribisala BS, Chen MJ, Mathers JC, Taylor R. Reversal of type 2 diabetes: normalisation of $\beta$ cell function in association with decreased pancreas and liver triacylglycerol. Diabetologia. 2011;54(10):2506-2514.

10. Jakicic JM. The effect of physical activity on body weight. Obesity (Silver Spring). 2009;17(suppl 3):S34-S38.

11. Dube JJ, Amati F, Stefanovic-Racic M, Toledo FG, Sauers SE, Goodpaster BH. Exercise-induced alterations in intramyocellular lipids and insulin resistance: the athlete's paradox revisited. Am J Physiol Endocrinol Metab. 2008;294(5):E882-E888.

12. Duncan GE, Perri MG, Theriaque DW, Hutson AD, Eckel RH, Stacpoole PW. Exercise training, without weight loss, increases insulin sensitivity and postheparin plasma lipase activity in previously sedentary adults. Diabetes Care. 2003;26(3):557-562.

13. Sigal RJ, et al. Effects of aerobic training, resistance training, or both on glycemic control in type 2 diabetes: a randomized trial. Ann Intern Med. 2007;147(6):357-369.

14. Hu FB, et al. Walking compared with vigorous physical activity and risk of type 2 diabetes in women: a prospective study. JAMA. 1999;282(15):1433-1439.

15. Manson JE, et al. A prospective study of walking as compared with vigorous exercise in the prevention of coronary heart disease in women. N EnglJMed.1999;341(9):650-658.

16. Devlin JT, Hirshman M, Horton ED, Horton ES. Enhanced peripheral and splanchnic insulin sensitivity in NIDDM men after single bout of exercise. Diabetes. 1987;36(4):434-439.

17. Nishida $Y$, et al. Effect of moderate exercise training on peripheral glucose effectiveness, insulin sensitivity, and endogenous glucose production in healthy humans estimated by a twocompartment-labeled minimal model. Diabetes. 2004;53(2):315-320.

18. Short KR, et al. Impact of aerobic exercise training on age-related changes in insulin sensitivity and muscle oxidative capacity. Diabetes. 2003;52(8):1888-1896.

19. Jakicic JM, Marcus BH, Lang W, Janney C. Effect of exercise on 24-month weight loss maintenance in overweight women. Arch Intern Med. 2008;168(14):1550-1559.

20. Shah M, et al. High-volume exercise program in obese bariatric surgery patients: a random ized, controlled trial. Obesity (Silver Spring). 2011;19(9):1826-1834.

21. Bond DS, et al. Objective quantification of physical activity in bariatric surgery candidates and normal-weight controls. Surg Obes Relat Dis. 2010;6(1):72-78.

22. Bond DS, Phelan S, Leahey TM, Hill JO, Wing RR. Weight-loss maintenance in successful weight losers: surgical vs non-surgical methods. Int JObes (Lond). 2009;33(1):173-180.

23. Klem ML, et al. A case-control study of successful maintenance of a substantial weight loss: individuals who lost weight through surgery versus those who lost weight through nonsurgical means. Int JObes Relat Metab Disord. 2000;24(5):573-579.

24. Bond DS, et al. Impact of self-reported physical activity participation on proportion of excess weight loss and BMI among gastric bypass surgery patients. Am Surg. 2004;70(9):811-814.

25 . Bond DS, et al. Becoming physically active after bariatric surgery is associated with improved weight loss and health-related quality of life. Obesity (Silver Spring). 2009;17(1):78-83.

26. Cook CM, Edwards C. Success habits of long-term gastric bypass patients. Obes Surg. 1999;9(1):80-82.

27. Silver HJ, Torquati A, Jensen GL, Richards WO. Weight, dietary and physical activity behaviors two years after gastric bypass. Obes Surg. 2006;16(7):859-864.

28. Wolfe BL, Terry ML. Expectations and outcomes with gastric bypass surgery. Obes Surg. 2006;16(12):1622-1629.

29. Bond DS, et al. Pre- to postoperative physical activity changes in bariatric surgery patients: self report vs. objective measures. Obesity (Silver Spring). 2010;18(12):2395-2397.

30. Wei M, et al. and Blair SN. Relationship between low cardiorespiratory fitness and mortality in normal-weight, overweight, and obese men. JAMA. 1999;282(16):1547-1553.

31. Bikman BT, et al. Mechanism for improved insulin sensitivity after gastric bypass surgery. J Clin Endocrinol Metab. 2008;93(12):4656-4663.

32. Goodpaster BH. Mitochondrial deficiency is associated with insulin resistance. Diabetes. 2013;62(4):1032-1035.

33. Best JD, Kahn SE, Ader M, Watanabe RM, Ni TC, Bergman RN. Role of glucose effectiveness in the determination of glucose tolerance. Diabetes Care. 1996;19(9):1018-1030.

34. Kahn SE, et al. The contribution of insulin-depen- dent and insulin-independent glucose uptake to intravenous glucose tolerance in healthy human subjects. Diabetes. 1994;43(4):587-592.

35. Schwartz MW, et al. Cooperation between brain and islet in glucose homeostasis and diabetes. Nature. 2013;503(7474):59-66.

36. Lorenzo C, et al. Disposition index, glucose effectiveness, and conversion to type 2 diabetes: the Insulin Resistance Atherosclerosis Study (IRAS). Diabetes Care. 2010;33(9):2098-2103.

37. Escalante-Pulido M, Escalante-Herrera A, MilkeNajar ME, Alpizar-Salazar M. Effects of weight loss on insulin secretion and in vivo insulin sensitivity in obese diabetic and non-diabetic subjects. Diabetes Nutr Metab. 2003;16(5-6):277-283.

38. Letiexhe MR, Scheen AJ, Gerard PL, Desaive C, Lefebvre PJ. Postgastroplasty recovery of ideal body weight normalizes glucose and insulin metabolism in obese women. JClin Endocrinol Metab. 1995;80(2):364-369.

39. Baillot A, Asselin M, Comeau E, Meziat-Burdin A, Langlois MF. Impact of excess skin from massive weight loss on the practice of physical activity in women. Obes Surg. 2013;23(11):1826-1834.

40. Wouters EJ, Larsen JK, Zijlstra H, van Ramshorst B, Geenen R. Physical activity after surgery for severe obesity: the role of exercise cognitions. Obes Surg. 2011;21(12):1894-1899.

41. Jackson AS, Sui X, Hebert JR, Church TS, Blair $\mathrm{SN}$. Role of lifestyle and aging on the longitudinal change in cardiorespiratory fitness. Arch Intern Med. 2009;169(19):1781-1787.

42. Farrell SW, Braun L, Barlow CE, Cheng YJ, Blair $\mathrm{SN}$. The relation of body mass index, cardiorespiratory fitness, and all-cause mortality in women. Obes Res. 2002;10(6):417-423.

43. Egberts K, Brown WA, Brennan L, O’Brien PE. Does exercise improve weight loss after bariatric surgery? A systematic review. Obes Surg. 2012;22(2):335-341.

44. Lichtman SW, et al. Discrepancy between self-reported and actual caloric intake and exercise in obese subjects. $\mathrm{N}$ Engl J Med. 1992;327(27):1893-1898.

45. Jakicic JM, Polley BA, Wing RR. Accuracy of self-reported exercise and the relationship with weight loss in overweight women. Med Sci Sports Exerc. 1998;30(4):634-638.

46. Bergman RN, Finegood DT, Ader M. Assessment of insulin sensitivity in vivo. Endocr Rev. 1985;6(1):45-86.

47. Boston RC, Stefanovski D, Moate PJ, Sumner AE, Watanabe RM, Bergman RN. MINMOD Millennium: a computer program to calculate glucose effectiveness and insulin sensitivity from the frequently sampled intravenous glucose tolerance test. Diabetes Technol Ther. 2003;5(6):1003-1015.

48. Goodpaster BH, Kelley DE, Wing RR, Meier A, Thaete FL. Effects of weight loss on regional fat distribution and insulin sensitivity in obesity. Diabetes. 1999;48(4):839-847.

49. American College of Sports Medicine., Whaley $\mathrm{MH}$, Brubaker PH, Otto RM, Armstrong LE. ACSM's Guidelines For Exercise Testing And Prescription. Philadelphia, Pennsylvania, USA: Lippincott Williams \& Wilkins; 2006. 